Richard S. Lemence, Dennis T. Leyson, Marian P. Roque/ TJMCS Vol .2 No.4 (2011) 607-618

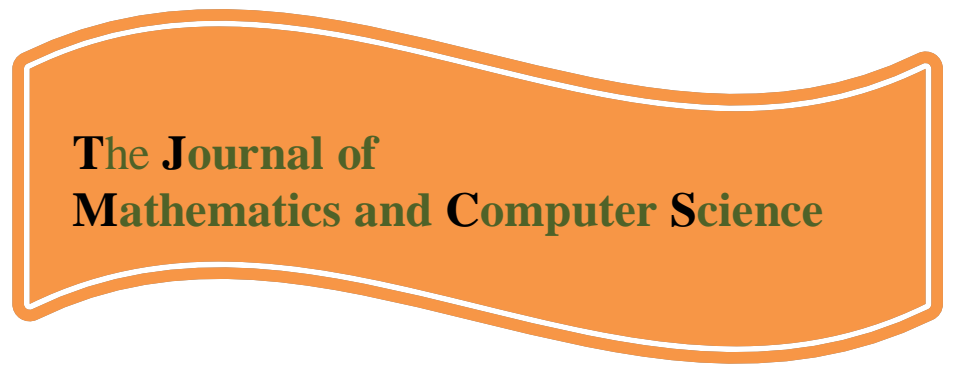

Available online at

\title{
http://www.TJMCS.com
}

The Journal of Mathematics and Computer Science Vol .2 No.4 (2011) 607-618

\section{On Some Geometric Properties of the Sphere $S^{n}$}

\author{
Richard S. Lemence ${ }^{*}$, Dennis T. Leyson ${ }^{2}$ and Marian P. Roque ${ }^{3}$ \\ Institute of Mathematics, College of Science, University of the Philippines, \\ Diliman, Quezon City, Philippines \\ 1rslemence@gmail.com, \\ 2dens_09@yahoo.com, \\ ${ }^{3}$ marian.roque11@gmail.com
}

Received: September 2010, Revised: December 2010

Online Publication: January 2011

\begin{abstract}
It is known that the sphere $S^{n}$ admits an almost complex structure only when $n=2$ or $n=6$. In this paper, we show that the sphere $S^{n}$ is a space of constant sectional curvature and using the results of T. Sato in [4], we determine the scalar curvature and the *-scalar curvature of $S^{6}$. We shall also prove that $S^{6}$ is a non-Kähler nearly Kähler manifold using the Levi-Civita connection on $S^{6}$ defined by H. Hashimoto and K. Sekigawa [3]. In [2], A. Gray and L. Hervella defined sixteen classes of almost Hermitian manifolds. We shall define quasi-Hermitian, a class of almost Hermitian manifolds and partially characterize almost Hermitian manifolds that belong to this class. Finally, under certain conditions, we shall show the sphere $S^{6}$ is quasi-Hermitian.
\end{abstract}

Keywords: Sphere, Kähler manifolds, Hermitian manifolds, quasiHermitian manifolds

AMS Subject Classification (MSC2010): 53B35, 53C55

\section{Preliminaries}

Let $M=(M, J, g)$ be a $2 n$-dimensional almost Hermitian manifold with the almost complex structure $J$ and Riemannian metric $g$. Let $\nabla$ be the Levi-Civita connection on $M$ and $R$ the Riemannian curvature tensor defined by

*Currently, he is a postdoctoral research fellow at the Academic Production, Ochanomizu University, Bunkyo-ku, Tokyo, Japan. 
Richard S. Lemence, Dennis T. Leyson, Marian P. Roque/ TJMCS Vol .2 No.4 (2011) 607-618

$$
R(X, Y) Z=\nabla_{X}\left(\nabla_{Y} Z\right)-\nabla_{Y}\left(\nabla_{X}\right) Z-\nabla_{[X, Y]} Z
$$

for $X, Y$ and $Z \in \mathfrak{X}(M)$, where $\mathfrak{X}(M)$ denotes the Lie algebra of all smooth vector fields on $M$.

If $U$ is a unit normal vector to $M$ and $V \in \mathfrak{X}(M)$, the shape operator of $M$ in $\mathbb{R}^{n+1}$, denoted $S(V)$, is defined by

$$
\begin{aligned}
S(V) & =-\nabla_{V}^{\mathbb{R}^{n+1}} U \\
& =-\sum_{i=1}^{n+1} \mathbf{V}\left[U^{i}\right] e_{i},
\end{aligned}
$$

where $e_{i}$ is the standard $i^{\text {th }}$ basis vector for $\mathbb{R}^{n+1}$ and $\mathbf{V}[\cdot]$ denotes the ordinary directional derivative $\mathbf{v}[f]=\nabla f \cdot \mathbf{V}$. For $X, Y, Z \in \mathfrak{X}(M)$,

$$
R(X, Y) Z=g(S(X), Z) S(Y)-g(S(Y), Z) S(X)
$$

The Ricci tensor $\rho$ is a symmetric tensor of type $(0,2)$ defined by

$$
\begin{aligned}
\rho(X, Y) & =\operatorname{trace}[Z \mapsto R(X, Z) Y] \\
& =\sum_{i=1}^{2 n} R\left(e_{i}, X, e_{i}, Y\right),
\end{aligned}
$$

where $\left\{e_{1}, \ldots, e_{2 n}\right\}$ is an arbitrary orthonormal basis for $T_{p}(M)$, the tangent space to $M$ at the point $p$. The Ricci tensor transformation $Q$ of type $(1,1)$ is given by

$$
\rho(X, Y)=g(Q X, Y)
$$

and the trace of $Q$ is called the scalar curvature $\tau$ of $R$. Furthermore, we denote by $\rho^{*}$ and $\tau^{*}$ the Ricci ${ }^{*}$-tensor and the ${ }^{*}$-scalar curvature on $M$, respectively. The tensor $\rho^{*}$ is defined pointwise by

$$
\begin{aligned}
\rho^{*}(X, Y) & =\operatorname{trace}(Z \mapsto R(J Z, X) J Y) \\
& =-\sum_{i=1}^{2 n} R\left(X, e_{i}, J Y, J e_{i}\right) \\
& =-\frac{1}{2} \sum_{i=1}^{2 n} R\left(X, J Y, e_{i}, J e_{i}\right)
\end{aligned}
$$


where $X, Y$ and $Z \$ \backslash$ in $T_{p}(M), R(X, Y, Z, W)=g(R(X, Y) Z, W)$ and $\left\{e_{i}\right\}$ is an orthonormal basis of $T_{p}(M)$. We also define analogously the Ricci *operator, denoted by $Q^{*}$, by $\rho^{*}(X, Y)=g\left(Q^{*} X, Y\right)$ for $X$ and $Y \in T_{p}(M)$. The trace of $Q^{*}$ is called the *-scalar curvature $\tau^{*}$ on $M$. We note that $\rho^{*}$ satisfies $\rho^{*}(J X, J Y)=\rho^{*}(Y, X)$ but is not symmetric in general.

An almost complex structure $J$ is said to be integrable if $M$ admits a complex structure and the derived almost complex structure coincides with $J$. We also say that the almost complex manifold $(M, J)$ is integrable if $J$ is integrable.

The Nijenhuis tensor $N$ of an almost complex structure $J$ is a tensor field of type $(1,2)$ defined by

$$
N(X, Y)=[J X, J Y]-J[J X, Y]-J[X, J Y]-[X, Y],
$$

for $X, Y \in \mathfrak{X}(M)$. In [5], B. Kruglikov showed that the Nijenhuis tensor $N$ can be expressed in terms of any symmetric connection $\nabla$ on $M$, i.e.,

$$
N(X, Y)=\left(\nabla_{X} J\right)(J Y)+\left(\nabla_{J X} J\right) Y-\left(\nabla_{Y} J\right)(J X)-\left(\nabla_{J Y} J\right) X .
$$

It is easy to verify that the Nijenhuis tensor satisfies the following properties:

$$
\begin{gathered}
N(X, Y)=-N(Y, X) \\
N(J X, Y)=-J N(X, Y) \\
N(X, J Y)=-J N(X, Y) .
\end{gathered}
$$

A. Newlander and L. Nirenberg, in [1], established the following result on the integrability of an almost complex structure $J$.

Theorem 1. An almost complex structure $J$ is integrable if and only if the Nijenhuis tensor $N$ vanishes on $M$.

As a consequence of the Newlander and Nirenberg above, we have the following corollary.

Corollary 1. Any 2-dimensional almost complex manifold $(M, J)$ is integrable.

Let $X$ and $Y \in T_{p}(M)$ such that $X$ and $Y$ are linearly independent. The sectional curvature $K_{\pi}$ of the 2-dimensional subspace $\pi$ of $T_{p}(M)$ spanned by $\{X, Y\}$ is given by 
Richard S. Lemence, Dennis T. Leyson, Marian P. Roque/ TJMCS Vol .2 No.4 (2011) 607-618

$$
K_{\pi}=K(X, Y)=\frac{g(R(X, Y) X, Y)}{g(X, X) g(Y, Y)-(g(X, Y))^{2}},
$$

while the holomorphic sectional curvature, $H(X)$, is the sectional curvature of the subspace of $T_{p}(M)$ spanned by $\{X, J X\}$, i.e.,

$$
H(X)=\frac{g(R(X, J X) X, J X)}{(g(X, X))^{2}-(g(X, J X))^{2}}=K(X, J X)
$$

An almost complex manifold $(M, J)$ equipped with a Riemannian metric $g$ that satisfies

$$
g(J X, J Y)=g(X, Y)
$$

for all $X, Y \in \mathfrak{X}(M)$, is called an almost Hermitian manifold, denoted by $(M, J, g)$. In [2], sixteen classes of almost Hermitian manifolds were defined by Gray and Hervella. The list includes Kähler, Hermitian and nearly Kähler manifolds. An almost Hermitian manifold $M$ is called a Kähler manifold if $\nabla J=0$, for all $X, Y \in \mathfrak{X}(M)$. It is called Hermitian if $N=0$. It is a nearly Kähler manifold if $\left(\nabla_{X} J\right) Y+\left(\nabla_{Y} J\right) X=0$, or equivalently $\left(\nabla_{X} J\right) X=0$, for all $X \in \mathfrak{X}(M)$.

\section{Geometry of $S^{n}$}

It is known that the standard sphere $S^{n}$ admits an almost complex structure only when $n=2$ or $n=6$. To construct an almost complex structure on $S^{2}$, we use the span of the quaternions. Let $H=\operatorname{span}_{\mathbb{R}}\{1, i, j, k\}$, where

$$
\begin{gathered}
i^{2}=j^{2}=k^{2}=-1 \\
i j=k, j k=i, k i=j \\
j i=-k, k j=-i, i k=-j .
\end{gathered}
$$

Then $\mathbb{R}^{3}$ can be identified with the set $\operatorname{Im} H=\operatorname{span}_{\mathbb{R}}\{i, j, k\}$. For $X=x^{1} i+x^{2} j+x^{3} k$ and $Y=y^{1} i+y^{2} j+y^{3} k \in \operatorname{Im} H$, the metric $g$ in $\operatorname{Im} H$ and the exterior product are defined as

$$
\begin{gathered}
g(X, Y)=x^{1} y^{1}+x^{2} y^{2}+x^{3} y^{3} \\
X \times Y=\left(x^{2} y^{3}-x^{3} y^{2}\right) i+\left(x^{3} y^{1}-x^{1} y^{3}\right) j+\left(x^{1} y^{2}-x^{2} y^{1}\right) k,
\end{gathered}
$$


and the sphere $S^{2}$ is given by $S^{2}=\{p \in \operatorname{Im} H \mid g(p, p)=1\}$. With this definition, the tangent space to $S^{2}$ at a point $p \in S^{2}$ is $T_{p} S^{2}=\{X \in \operatorname{Im} H \mid g(X, p)=0\}$.

Let $p=p^{1} i+p^{2} j+p^{3} k \in S^{2}$ and $X=x^{1} i+x^{2} j+x^{3} k \in T_{p} S^{2}$. Define a tensor $J_{p}: T_{p} S^{2} \rightarrow T_{p}\left(S^{2}\right)$ by

$$
J_{p} X=X \times p=\left(x^{2} p^{3}-x^{3} p^{2}\right) i+\left(x^{3} p^{1}-x^{1} p^{3}\right) j+\left(x^{1} p^{2}-x^{2} p^{1}\right) k
$$

This $J_{p}$ induces a tensor $J$ on $S^{2}$ such that $J^{2}=-I$, hence the following theorem.

Theorem 2. The sphere $S^{2}$ is an almost complex manifold.

Theorem 3. The sphere $S^{2}$ is an almost Hermitian manifold.

Proof. Let $X=x^{1} i+x^{2} j+x^{3} k$ and $Y=y^{1} i+y^{2} j+y^{3} k \in T_{p}\left(S^{2}\right)$. Then, for any $p \in S^{2}$, we have

$$
\begin{gathered}
g\left(J_{p} X, J_{p} Y\right)=\left(x^{2} p^{3}-x^{3} p^{2}\right)\left(y^{2} p^{3}-y^{3} p^{2}\right)+\left(x^{3} p^{1}-x^{1} p^{3}\right)\left(y^{3} p^{1}-y^{1} p^{3}\right) \\
+\left(x^{1} p^{2}-x^{2} p^{1}\right)\left(y^{1} p^{2}-y^{2} p^{1}\right) \\
=x^{2} y^{2}\left(p^{3}\right)^{2}-x^{3} p^{3} y^{2} p^{2}-x^{2} p^{2} y^{3} p^{3}+x^{3} y^{3}\left(p^{2}\right)^{2} \\
+x^{3} y^{3}\left(p^{1}\right)^{2}-x^{1} p^{1} y^{3} p^{3}-x^{3} p^{3} y^{1} p^{1}+x^{1} y^{1}\left(p^{3}\right)^{2} \\
+x^{1} y^{1}\left(p^{2}\right)^{2}-x^{2} p^{2} y^{1} p^{1}-x^{1} p^{1} y^{2} p^{2}+x^{2} y^{2}\left(p^{1}\right)^{2} .
\end{gathered}
$$

Regrouping terms, we get

$$
\begin{aligned}
g\left(J_{p} X, J_{p} Y\right)= & x^{1} y^{1}\left(p^{1}\right)^{2}+x^{1} y^{1}\left(p^{3}\right)^{2}+x^{2} y^{2}\left(p^{1}\right)^{2}+x^{2} y^{2}\left(p^{3}\right)^{2} \\
& +x^{3} y^{3}\left(p^{1}\right)^{2}+x^{3} y^{3}\left(p^{2}\right)^{2}-x^{1} p^{1} y^{2} p^{2}-x^{1} p^{1} y^{3} p^{3} \\
& -x^{2} p^{2} y^{1} p^{1}-x^{2} p^{2} y^{3} p^{3}-x^{3} p^{3} y^{1} p^{1}-x^{3} p^{3} y^{2} p^{2} .
\end{aligned}
$$

Adding and subtracting $x^{1} y^{1}\left(p^{1}\right)^{2}+x^{2} y^{2}\left(p^{2}\right)^{2}+x^{3} y^{3}\left(p^{3}\right)^{2}$ will yield

$$
\begin{aligned}
g\left(J_{p} X, J_{p} Y\right)= & x^{1} y^{1}\left(p^{1}\right)^{2}+x^{1} y^{1}\left(p^{2}\right)^{2}+x^{1} y^{1}\left(p^{3}\right)^{2}+x^{2} y^{2}\left(p^{1}\right)^{2}+x^{2} y^{2}\left(p^{2}\right)^{2}+x^{2} y^{2}\left(p^{3}\right)^{2} \\
& +x^{3} y^{3}\left(p^{1}\right)^{2}+x^{3} y^{3}\left(p^{2}\right)^{2}+x^{3} y^{3}\left(p^{3}\right)^{2}-x^{1} p^{1} y^{1} p^{1}-x^{1} p^{1} y^{2} p^{2}-x^{1} p^{1} y^{3} p^{3} \\
& -x^{2} p^{2} y^{1} p^{1}-x^{2} p^{2} y^{2} p^{2}-x^{2} p^{2} y^{3} p^{3}-x^{3} p^{3} y^{1} p^{1}-x^{3} p^{3} y^{2} p^{2}-x^{3} p^{3} y^{3} p^{3} \\
= & x^{1} y^{1} g(p, p)+x^{2} y^{2} g(p, p)+x^{3} y^{3} g(p, p)-x^{1} p^{1} g(Y, p) \\
& -x^{2} p^{2} g(Y, p)-x^{3} p^{3} g(Y, p) \\
= & x^{1} y^{1}+x^{2} y^{2}+x^{3} y^{3}
\end{aligned}
$$




$$
=g(X, Y)
$$

From Corollary 1 and Theorem 2, the Nijenhuis tensor on $S^{2}$ vanishes. Combining this result with Theorem 3 and the definition of a Hermitian manifold, we have the following.

Theorem 4. The sphere $S^{2}$ is a Hermitian manifold.

Before we can define the almost complex structure on the unit sphere $S^{6}$, we recall first the Cayley algebra.Let $\mathcal{C}=\operatorname{span}_{\mathbb{R}}\{1, i, j, k, l, i l, j l, k l\}$ such that

$$
i^{2}=j^{2}=k^{2}=l^{2}=i l^{2}=j l^{2}=k l^{2}=-1,
$$

and if $e_{0}=1, e_{1}=i, e_{2}=j, e_{3}=k, e_{4}=l, e_{5}=i l, e_{6}=j l$ and $e_{7}=k l$, then Table 1 shows the multiplication of the basis elements of the Cayley algebra.

\begin{tabular}{|c|c|c|c|c|c|c|c|c|}
\hline & $e_{0}$ & $e_{1}$ & $e_{2}$ & $e_{3}$ & $e_{4}$ & $e_{5}$ & $e_{6}$ & $e_{7}$ \\
\hline$e_{0}$ & $e_{0}$ & $e_{1}$ & $e_{2}$ & $e_{3}$ & $e_{4}$ & $e_{5}$ & $e_{6}$ & $e_{7}$ \\
\hline$e_{1}$ & $e_{1}$ & $-e_{0}$ & $e_{3}$ & $-e_{2}$ & $e_{5}$ & $-e_{4}$ & $-e_{7}$ & $e_{6}$ \\
\hline$e_{2}$ & $e_{2}$ & $-e_{3}$ & $-e_{0}$ & $e_{1}$ & $e_{6}$ & $e_{7}$ & $-e_{4}$ & $-e_{5}$ \\
\hline$e_{3}$ & $e_{3}$ & $e_{2}$ & $-e_{1}$ & $-e_{0}$ & $e_{7}$ & $-e_{6}$ & $e_{5}$ & $-e_{4}$ \\
\hline$e_{4}$ & $e_{4}$ & $-e_{5}$ & $-e_{6}$ & $-e_{7}$ & $-e_{0}$ & $e_{1}$ & $e_{2}$ & $e_{3}$ \\
\hline$e_{5}$ & $e_{5}$ & $e_{4}$ & $-e_{7}$ & $e_{6}$ & $-e_{1}$ & $-e_{0}$ & $-e_{3}$ & $e_{2}$ \\
\hline$e_{6}$ & $e_{6}$ & $e_{7}$ & $e_{4}$ & $-e_{5}$ & $-e_{2}$ & $e_{3}$ & $-e_{0}$ & $-e_{1}$ \\
\hline$e_{7}$ & $e_{7}$ & $-e_{6}$ & $e_{5}$ & $e_{4}$ & $-e_{3}$ & $-e_{2}$ & $e_{1}$ & $-e_{0}$ \\
\hline
\end{tabular}

Table 1. Multiplication table of the basis elements of $\mathcal{C}$

Let $X, Y \in \mathcal{C}$. We define the metric $g$ (inner product) and the exterior product, respectively, as

$$
\begin{gathered}
g(X, Y)=-(\text { real part of } X Y) \\
X \times Y=\text { imaginary part of } X Y
\end{gathered}
$$

where $X Y$ is the product of $X$ and $Y$ in $\mathcal{C}$.

Remark. For any $X, Y, Z \in \mathcal{C}$, the inner and exterior products satisfy the following:

(i) $X \times Y=-(Y \times X)$ 
(ii) $g(X \times Y, Z)=g(X, Y \times Z)$

(iii) $X \times(Y \times Z)=g(X, Z) Y-g(X, Y) Z$

Notice that each element $z$ of $\mathcal{C}$ can be expressed as $z=\sum_{i=0}^{7} a^{i} e_{i}$. Here, we call the number $a^{0}$ the real part of $z$ and $\sum_{i=1}^{7} a^{i} e_{i}$ as its imaginary part. Denote the set of imaginary parts of elements of $\mathcal{C}$ by $\operatorname{ImC}$. Let $p\left(x^{1}, \ldots, x^{7}\right) \in \mathfrak{R}^{7}$. If we denote by $V_{p}$ the vector in $\mathbb{R}^{7}$ determined by the point $p$ with the origin, then $V_{p} \in \operatorname{ImC}$, i.e.,

$$
V_{p}=x^{1} e_{1}+x^{2} e_{2}+x^{3} e_{3}+x^{4} e_{4}+x^{5} e_{5}+x^{6} e_{6}+x^{7} e_{7}
$$

This means that $\mathfrak{R}^{7}$ can be identified with $\operatorname{ImC}$. Now observe that

$$
\begin{aligned}
g\left(V_{p}, V_{p}\right)=1 & \Leftrightarrow-\left\{\text { real part of }\left(V_{p} V_{p}\right)\right\}=1 \\
& \Leftrightarrow-\left(-\left(x^{1}\right)^{2}-\left(x^{2}\right)^{2}-\left(x^{3}\right)^{2}-\left(x^{4}\right)^{2}-\left(x^{5}\right)^{2}-\left(x^{6}\right)^{2}-\left(x^{7}\right)^{2}\right)=1 \\
& \Leftrightarrow\left(x^{1}\right)^{2}+\left(x^{2}\right)^{2}+\left(x^{3}\right)^{2}+\left(x^{4}\right)^{2}+\left(x^{5}\right)^{2}+\left(x^{6}\right)^{2}+\left(x^{7}\right)^{2}=1 \\
& \Leftrightarrow p \in S^{6} .
\end{aligned}
$$

Thus, we can define $S^{6}$ as

$$
S^{6}=\left\{V_{p} \in \operatorname{ImC} \mid g\left(V_{p}, V_{p}\right)=1\right\} .
$$

Since the tangent space at a point $p \in S^{6}$ is the set of all vectors orthogonal to $V_{p}$, then

$$
T_{p} S^{6}=\left\{X \in \operatorname{ImC} \mid g\left(X, V_{p}\right)=0\right\} .
$$

Let us define a tensor $J_{p}$ from $T_{p} S^{6}$ to $T_{p} S^{6}$ by

$$
J_{p} X=X \times V_{p}
$$

for $X \in T_{p} S^{6}$ and $p \in S^{6}$. Then

$$
\begin{aligned}
J_{p}^{2} X & =J_{p}\left(J_{p} X\right) \\
& =J_{p}\left(X \times V_{p}\right) \\
& =\left(X \times V_{p}\right) \times V_{p}
\end{aligned}
$$


Richard S. Lemence, Dennis T. Leyson, Marian P. Roque/ TJMCS Vol .2 No.4 (2011) 607-618

$$
\begin{aligned}
& =-V_{p} \times\left(X \times V_{p}\right) \\
& =-\left[g\left(V_{p}, V_{p}\right) X-g\left(V_{p}, X\right) V_{p}\right] \\
& =-X .
\end{aligned}
$$

So, $J_{p}$ induces a tensor $J$ such that $J^{2}=-I$, where $I$ is the identity map. Also,

Observe that

$$
g\left(J_{p} X, J_{p} Y\right)=g\left(X \times V_{p}, Y \times V_{p}\right)=g\left(X, V_{p} \times\left(Y \times V_{p}\right)\right) .
$$

$$
V_{p} \times\left(Y \times V_{p}\right)=g\left(V_{p}, V_{p}\right) Y-g\left(V_{p}, Y\right) V_{p}=Y
$$

Therefore,

$$
g\left(J_{p} X, J_{p} Y\right)=g(X, Y)
$$

This proves the following theorem.

Theorem 5. The sphere $S^{6}$ is an almost Hermitian manifold.

H. Hashimoto and K. Sekigawa [3] derived the Levi-Civita connection on $S^{6}$ and obtained

$$
\left(\nabla_{X} J\right) Y=-X \times Y+g\left(X \times Y, V_{p}\right) V_{p}
$$

for any $X, Y \in T_{p} S^{6}, p \in S^{6}$. One can check that this linear connection is not always zero. But, for any $X \in T_{p} S^{6}$, we have

$$
\left(\nabla_{X} J\right) X=-X \times X+g\left(X \times X, V_{p}\right) V_{p}=0 .
$$

Hence, we have the following theorem.

Theorem 6. The sphere $S^{6}$ is a non-Kähler nearly Kähler manifold.

We are now interested with the different curvature tensors on $S^{n}$. Let us determine first what the shape operator does with every tangent vector to $S^{n}$. In coordinates, the unit normal vector to $S^{n}$ at a point $\left(x^{1}, \ldots, x^{n+1}\right)$ is given by $U=\left(x^{1}, \ldots, x^{n+1}\right)$. Let $V=\left(V^{1}, \ldots, V^{n+1}\right)$ be any tangent vector to $S^{n}$. The covariant derivative is the coordinate-wise directional derivative. So,

$$
S(V)=-\nabla_{V} U=-\left(V\left[x^{1}\right], \ldots, V\left[x^{n+1}\right]\right) .
$$


Richard S. Lemence, Dennis T. Leyson, Marian P. Roque/ TJMCS Vol .2 No.4 (2011) 607-618

But for $i=1, \ldots, n+1$,

$$
\begin{aligned}
V\left[x^{i}\right] & =\sum_{j=1}^{n+1} \frac{\partial x^{i}}{\partial x^{j}} V^{j} \\
& =V^{j} \delta_{i}^{j} \\
& =V^{i} .
\end{aligned}
$$

Therefore,

$$
S(V)=-\left(V^{1}, \ldots, V^{n+1}\right)=-V
$$

Now, let $X$ and $Y$ be orthonormal tangent basis vectors at $p \in S^{n}$, i.e.,

Then

$$
\begin{aligned}
& g(X, X)=g(Y, Y)=1 \\
& g(X, Y)=g(Y, X)=0 .
\end{aligned}
$$

$$
\begin{aligned}
R(X, Y) X & =g(S(X), X) S(Y)-g(S(Y), X) S(X) \\
& =g(-X, X)(-Y)-g(-Y, X)(-X) \\
& =g(X, X) Y-g(Y, X) X \\
& =Y .
\end{aligned}
$$

Solving for the sectional curvature, we get

$$
\begin{aligned}
K(X, Y) & =\frac{g(R(X, Y) X, Y)}{g(X, X) g(Y, Y)-(g(X, Y))^{2}} \\
& =\frac{g(Y, Y)}{g(X, X) g(Y, Y)-(g(X, Y))^{2}} \\
& =1 .
\end{aligned}
$$

Theorem 7. The sphere $S^{n}$ is a space of constant sectional curvature with $K(X, Y)=1$ for $X, Y \in T_{p} S^{n}$ and for all $p \in S^{n}$.

As stated earlier, $S^{2}$ and $S^{6}$ both admit an almost complex structure $J$. Hence, we have the following results.

Corollary 2. The unit spheres $S^{2}$ and $S^{6}$ are spaces of constant holomorphic sectional curvature with $H(X)=1$, for any nonzero tangent vector $X$.

Proof. For any nonzero tangent vector $X$, 
Richard S. Lemence, Dennis T. Leyson, Marian P. Roque/ TJMCS Vol .2 No.4 (2011) 607-618

$$
H(X)=K(X, J X)=1
$$

In [4], T. Sato showed that if $M$ is a non-Kähler nearly Kähler manifold of dimension $n$ with pointwise constant holomorphic sectional curvature $H(X)=c(p)$, then

$$
\tau=\frac{5 n(n+2) c(p)}{8}
$$

and

$$
\tau+3 \tau^{*}=n(n+2) c(p)
$$

We now have the following result.

Theorem 8. The sphere $S^{6}$ being a non-Kähler nearly Kähler manifold with sectional curvature 1, its scalar curvature $\tau$ and ${ }^{*}$-scalar curvature $\tau *$ are

$$
\tau=30 \text { and } \tau^{*}=6
$$

Proof.

$$
\tau=\frac{5(6)(6+2)}{8}=30
$$

and

$$
30+3 \tau *=6(8) \Rightarrow \tau *=\frac{6(8)-30}{3}=6
$$

It is interesting to note that in the 6-dimensional unit sphere $S^{6}$, the Ricci *tensor $\rho^{*}$ is a conformal of the Ricci tensor $\rho$, i.e., $\rho^{*}=\frac{1}{5} \rho$. T. Sato also proved the following theorems for the 6-dimensional case.

Theorem 9. There does not exist any dimensional, except 6-dimensional, non-Kähler nearly Kähler manifold of pointwise constant holomorphic sectional curvature.

Theorem 10. If $M$ be a non-Kähler nearly Kähler manifold of pointwise constant holomorphic sectional curvature then $M$ is locally isometric to a 6-dimensional sphere $S^{6}$.

In [2], A. Gray and L. Hervella defined sixteen classes of almost Hermitian manifolds based on linear invariants. We now define a class of almost Hermitian manifolds. Our definition of this class is based on both linear invariants and the exterior product. 
Richard S. Lemence, Dennis T. Leyson, Marian P. Roque/ TJMCS Vol .2 No.4 (2011) 607-618

Definition 1. An almost Hermitian manifold $\$(M, J, g) \$$ is called quasi-Hermitian if it satisfies

$$
X \times J_{p} Y+J_{p} X \times Y=0,
$$

for all $X, Y \in T_{p}(M), p \in M$.

With this definition, we have the following results.

Theorem 11. Any 2-dimensional almost Hermitian manifold is quasi-Hermitian.

Proof. Suppose $M$ is a 2-dimensional almost-Hermitian manifold. Let $X \in T_{p} M$, such that $X$ is nonzero. Then $\left\{X, J_{p} X\right\}$ is a local frame. Thus,

$$
\begin{aligned}
X \times J_{p} Y+J_{p} X \times Y & =X \times J_{p}\left(J_{p} X\right)+J_{p} X \times J_{p} X \\
& =X \times J_{p}^{2} X \\
& =X \times(-X) \\
& =0 .
\end{aligned}
$$

Theorem 12. The 6-dimensional sphere $S^{6}$ is Hermitian if and only if it is quasiHermitian.

Proof. Let $X, Y \in T_{p} S^{6}, p \in S^{6}$. Since the Levi-Civita connection is torsion-free, we have

$$
\begin{aligned}
N(X, Y)= & \left(\nabla_{X} J\right)(J Y)+\left(\nabla_{J X} J\right) Y-\left(\nabla_{Y} J\right)(J X)-\left(\nabla_{J Y} J\right) X \\
= & {\left[-X \times J_{p} Y+g\left(X \times J_{p} Y, V_{p}\right) V_{p}\right]+\left[-J_{p} X \times Y+g\left(J_{p} X \times Y, V_{p}\right) V_{p}\right] } \\
& -\left[-Y \times J_{p} X+g\left(Y \times J_{p} X, V_{p}\right) V_{p}\right]-\left[-J_{p} Y \times X+g\left(J_{p} Y \times X, V_{p}\right) V_{p}\right] \\
= & \left(-X \times J_{p} Y\right)-\left(J_{p} X \times Y\right)+\left(Y \times J_{p} X\right)+\left(J_{p} Y \times X\right)+g\left(X \times J_{p} Y, V_{p}\right) V_{p} \\
+ & g\left(J_{p} X \times Y, V_{p}\right) V_{p}-g\left(Y \times J_{p} X, V_{p}\right) V_{p}-g\left(J_{p} Y \times X, V_{p}\right) V_{p} \\
= & -\left(X \times J_{p} Y\right)-\left(J_{p} X \times Y\right)-\left(J_{p} X \times Y\right)-\left(X \times J_{p} Y\right)+g\left(X \times J_{p} Y, V_{p}\right) V_{p} \\
& +g\left(J_{p} X \times Y, V_{p}\right) V_{p}-g\left(Y \times J_{p} X, V_{p}\right) V_{p}-g\left(J_{p} Y \times X, V_{p}\right) V_{p} \\
= & -2\left(X \times J_{p} Y+J_{p} X \times Y\right)+g\left(X \times J_{p} Y, V_{p}\right) V_{p}+g\left(J_{p} X \times Y, V_{p}\right) V_{p} \\
& -g\left(Y \times J_{p} X, V_{p}\right) V_{p}-g\left(J_{p} Y \times X, V_{p}\right) V_{p} \\
= & -2\left(X \times J_{p} Y+J_{p} X \times Y\right)-g(X, Y) V_{p} \\
& +\mathrm{g}(\mathrm{X}, \mathrm{Y}) \mathrm{V}_{-} \mathrm{p}+\mathrm{g}(\mathrm{X}, \mathrm{Y}) \mathrm{V}_{-} \mathrm{p}-\mathrm{g}(\mathrm{X}, \mathrm{Y}) \mathrm{V}_{-} \mathrm{p} \\
= & -2\left(X \times J_{p} Y+J_{p} X \times Y\right) .
\end{aligned}
$$

Hence, 
Richard S. Lemence, Dennis T. Leyson, Marian P. Roque/ TJMCS Vol .2 No.4 (2011) 607-618

$$
N(X, Y)=0 \text { if and only if } X \times J_{p} Y+J_{p} X \times Y=0
$$

Acknowledgment. We would like to thank the referees for their comments and suggestions.

\section{References}

[1] A. Newlander and L. Nirenberg, Complex Analytic Coordinates in Almost Com- plex Manifolds, Annals of Mathematics, Vol. 2, No.65, 1957, 391-404

[2] A. Gray and L.M. Hervella, The sixteen classes of almost Hermitian manifolds and their linear invariants, Annali di Matematica, Vol. 123, No. 4, 1980, 35-58

[3] H. Hashimoto and K. Sekigawa, Submanifolds of a nearly-Kähler 6dimensional sphere, Proceedings of the Eighth International Workshop on Differential Geom- etry, Vol. 8, 2004, 23-45

[4] T. Sato, Curvatures of almost Hermitian manifolds, Unpublished

[5] B. Kruglikov, Nijenhuis tensors and obstructions for pseudoholomorphic map- ping constructions, Matematical Notes 63, Vol. 4, 1998, 541-561 